\title{
Zum Verhältnis herkunftsrechtlicher Kennzeichnungs- zu lebensmittelrechtlichen Deklarationsvorschriften
}

Kommentar zum Urteil des Bundesgerichts 2C_761/2017 vom 25. Juni 2018 (Lozärner Bier)

Jürg Simon *

Wieviel «Lozärn» muss ein einem «Lozärner Bier» drin sein? Im Urteil 2C_761/2017 vom 25. Juni 2018 hat das Bundesgericht erstmals seit dem Inkraftreten der Revision des MSchG und des LMG das Verhältnis herkunftsrechtlicher Kennzeichnungs- zu lebensmittelrechtlichen Deklarationsvorschriften erörtert. Es hat dabei bestätigt, dass lebensmittelrechtlich verpflichtende deklaratorische Angaben auch die Vorschriften von Art. $47 f$. MSchG über die Warenherkunft einhalten müssen, sofern diese Angaben kennzeichnend eingesetzt werden. Umgekehrt dürfen Lebensmittel, obwohl die Herkunftskriterien nach Art. $47 f$. MSchG dies allenfalls sogar erlauben würden, nicht so aufgemacht und gekennzeichnet werden, dass Konsumenten getäuscht werden, weil bei ihnen aufgrund der Kennzeichnung tatsachenwidrige Vorstellungen geweckt werden. Für die Beurteilung einer Produktgestaltung ist das gesamte Erscheinungsbild des Produkts beim Durchschnittskonsumenten massgebend. Dieses wird massgeblich von den charakteristischen Eigenschaften eines Produkts geprägt.

I. Einführung

II. Sachverhalt

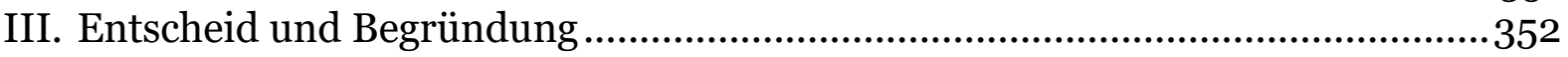

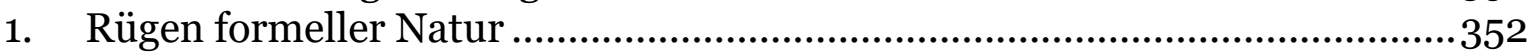

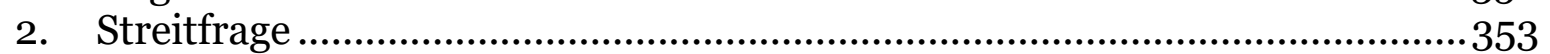

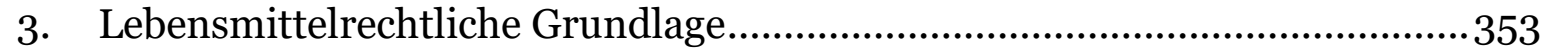

4. Verhältnis des Lebensmittelrechts zum Herkunftskennzeichenrecht ............354

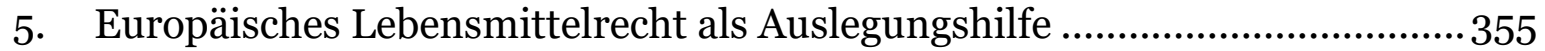

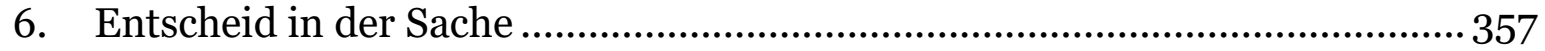

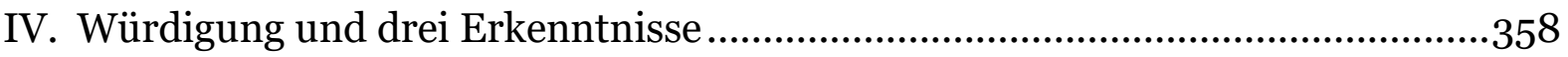

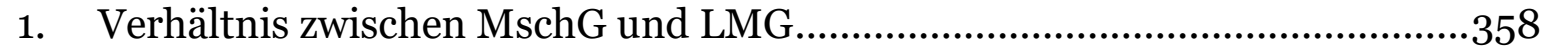

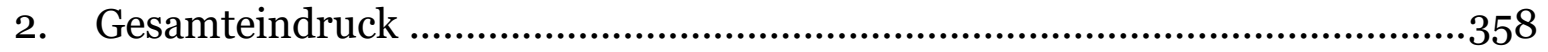

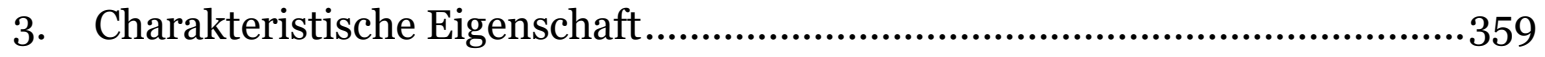

Zitiervorschlag: Jürg Simon, Zum Verhältnis herkunftsrechtlicher Kennzeichnungszu lebensmittelrechtlichen Deklarationsvorschriften, in: sui-generis 2018, S. 350

URL: $\quad$ sui-generis.ch/79

DOI: $\quad$ https://doi.org/10.21257/sg.79

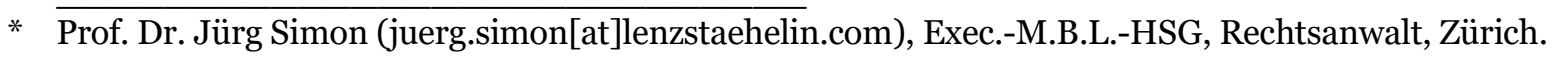




\section{Einführung}

1 Im Jahr 2017 sind sowohl die revidierte Fassung des Markenschutzgesetzes ${ }^{1}$ wie jene des Lebensmittelgesetzes ${ }^{2}$ in Kraft getreten. Die beiden Erlasse überschneiden sich im Bereich des Täuschungsschutzes bei der Verwendung von geographischen Angaben (Herkunftsangaben im MSchG; Deklaration z.B. des Produktionslandes im LMG, irreführende Aufmachungen). Seit 1. Mai 2017 enthält Art. 18 Abs. 2 Satz 2 LMG eine ausdrückliche Koordinationsbestimmung für das Verhältnis zwischen MSchG und LMG; diese ordnet an, dass die Bestimmungen des MSchG über Angaben zur schweizerischen Herkunft vorbehalten bleiben.

Im vorliegenden Fall 3 «Lozärner Bier» versuchte die Beschwerdeführerin, den von den Vorinstanzen lebensmittelrechtlich begründeten Täuschungsvorwurf mit marken- bzw. herkunftsrechtlichen Kriterien zu entkräften. Dies zwang das Bundesgericht, das Verhältnis zwischen Lebensmittelrecht und Marken- bzw. Herkunftsrecht erstmals nach den Revisionen von MSchG und LMG grundlegend zu erörtern. Es musste zudem insbesondere die Frage klären, ob und allenfalls wie sich das Schutzniveau des neuen LMG aufgrund seiner wesentlichen Orientierung am EU-Recht verändert hat. Schliesslich ergriff es die Gelegenheit und fasste die Grundsätze des lebensmittelrechtlichen Täuschungsschutzes geradezu lehrbuchmässig zusammen.

Bundesgesetz über den Schutz von Marken und Herkunftsangaben vom 28. August 1992 (Markenschutzgesetz, MSchG; SR 232.11).

2 Bundesgesetz über Lebensmittel und Gebrauchsgegenstände vom 20. Juni 2014 (Lebensmittelgesetz, LMG; SR 817.0).

3 Urteil des Bundesgerichts 2C_761/2017 vom 25. Juni 2018, zur Publikation vorgesehen.

\section{Sachverhalt}

3 Die Lozärner Bier AG bezweckt insbesondere die Herstellung und den Vertrieb von Bier, namentlich der Marke «Lozärner Bier»4, und von weiteren Getränken und Lebensmitteln. Sie verfügt über keine eigene Brauerei. Ihre Geschäftsräumlichkeiten befinden sich am statutarischen Sitz der Gesellschaft in Luzern.

4 Im Rahmen lebensmittelrechtlicher Kontrollen beanstandete die Dienststelle Lebensmittelkontrolle und Verbraucherschutz des Kantons Luzern (DILV), dass eine Produktprobe «Lozärner Bier Lager» keine Kennzeichnung aufweise und dass dieses Bier ausserhalb des Kantons Luzern (offenbar im Kanton Schaffhausen) von einer anderen Brauerei hergestellt und abgefüllt werde. Dieses Bier wurde vertrieben in Dosen in blauweisser Aufmachung, sowohl in Farbe wie Geometrie dem Luzerner Wappen nachempfunden.

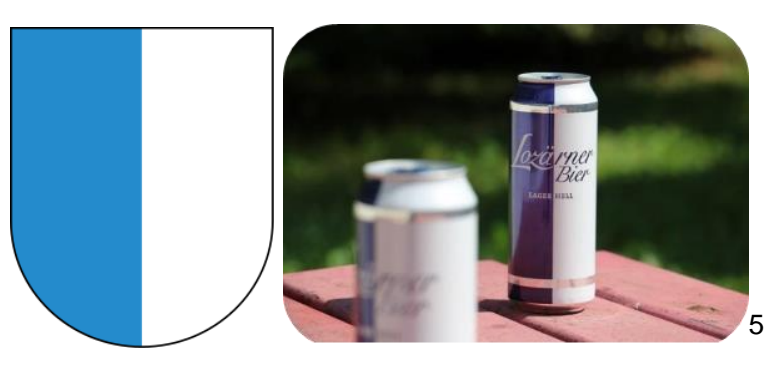

4 Die Marke CH 649'462 Lozärner Bier (fig.) ist u.a. eingetragen für Bier schweizerischer Herkunft und andere alkoholische Getränke luzernischer Herkunft. Das Eidgenössische Institut für Geistige Eigentum (IGE) hatte im Rahmen der Markeneintragung bei der Prüfung der absoluten Ausschlussgründe nach Art. 2 MSchG offenbar die Herkunftserwartung der Konsumenten von Bier und von anderen alkoholischen Getränken (z.B. Wein) differenziert beurteilt.

5 Bild: Christian Volken/luzernerzeitung.ch, Luzern, 20. Juli 2017, siehe bspw. Stefan Dähler, URTEIL: Etikettenschwindel: Kalte Dusche für «Lozärner Bier», luzernerzeitung.ch vom 20. Juli 2017. 
Rückseite der Dose enthielt den Vermerk «Lozärner Bier AG, Business Park Luzern, Littauerboden 1, CH-6014 Luzern». Ebenfalls auf der Dosenrückseite angebracht war ein Landesumriss der Schweiz und darüber der Vermerk «hergestellt und abgefüllt in der Schweiz». Die DILV beanstandete die Probe als täuschend im Sinne von Art. 10 der Lebensmittel- und Gebrauchsgegenständeverordnung vom 23. November $2005^{6}$ und verpflichtete die Lozärner Bier AG mittels Verfügung vom 6. September 2016 unter anderem, Massnahmen zu treffen, damit das Produkt «Lozärner Bier Lager» (Probe Nr. xy) die lebensmittelrechtlichen Anforderungen erfüllt, sowie den zeitlichen Rahmen für die Umsetzung dieser Massnahmen mitzuteilen. Die Einsprache der Lozärner Bier AG gegen diese Verfügung sowie die Verwaltungsgerichtsbeschwerde an das Kantonsgericht des Kantons Luzern waren erfolglos.

\section{Entscheid und Begründung}

\section{Rügen formeller Natur}

6 Das Bundesgericht hatte zunächst Rügen formeller Natur zu behandeln. Dazu gehörte u.a. das Argument der Lozärner Bier AG (Beschwerdeführerin), sie sei nicht zur Stellungnahme zu einem allfälligen Einfluss des am 1. Mai 2017 in Kraft getretenen neuen Lebensmittelrechts eingeladen worden. Damit sei ihr Anspruch auf rechtliches Gehört verletzt worden.
$6 \overline{\text { AS } 2005 \text { 5451; fortan: aLGV; gemäss Art. } 94}$ Abs. 1 i.V.m. Art. 96 der Lebensmittel- und Gebrauchsgegenständeverordnung vom 16. Dezember 2016 (LGV; SR 817.02) in Kraft bis 30. April 2017.
7 Das Bundesgericht weist hierzu auf seine ständige Rechtsprechung hin, wonach ein solcher Anspruch nur besteht, wenn (a) eine Behörde ihren Entscheid mit einer Rechtsnorm oder einem Rechtstitel zu begründen beabsichtigt, der im bisherigen Verfahren nicht herangezogen wurde, (b) auf den sich die Parteien nicht berufen haben und (c) mit dessen Erheblichkeit sie im konkreten Fall nicht rechnen konnten. Entscheidend sei, dass es der betroffenen Partei ermöglicht wurde, ihren Standpunkt wirksam zur Geltung zu bringen (E. 3.1.1).

8 Die Vorinstanz hatte im konkreten Fall durchaus erwogen, dass die lebensmittelrechtliche Gesetzgebung im Hinblick auf die Angleichung des schweizerischen Lebensmittelrechts an das Recht der Europäischen Union (EU) per 1. Mai 2017 vollständig revidiert wurde. Sie ging davon aus, die Frage nach dem intertemporal anwendbaren Recht sei indes nicht entscheiderheblich, weil die massgeblichen Bestimmungen des neuen und alten Rechts inhaltlich deckungsgleich seien. Das Bundesgericht schliesst daraus, dass die Beschwerdeführerin sich im bisherigen Verfahren ohne weiteres zu den relevanten Bestimmungen habe äussern können. Es weist ergänzend ausdrücklich darauf hin, dass die seit 1. Mai 2017 geltende Lebensmittelgesetzgebung unabhängig vom hier zu beurteilenden Fall auf den von der Beschwerdeführerin verfolgten Zweck «Vertrieb von Bieren» grundsätzlich Anwendung finde. Bei der Beschwerdeführerin dürften indes «Kenntnisse über wesentliche Rechtsänderungen in diesem Bereich, der ihr Kerngeschäft in zentralen Punkten betrifft», vorausgesetzt werden. Sogar wenn das neue Recht für die Beschwerdeführerin günstiger wäre, 
habe diese von der Vorinstanz nicht auf die Rechtsänderung aufmerksam gemacht werden und dazu zur Stellungnahme eingeladen werden müssen. Die Vorinstanz habe bei der Beschwerdeführerin in der Tat Kenntnis über das Inkrafttreten des neuen Lebensmittelrechts voraussetzen dürfen. Es wäre dieser freigestanden, von sich aus im vorinstanzlichen Verfahren zu den neuen Bestimmungen Stellung zu nehmen.

\section{Streitfrage}

9 In der Sache selbst vertraten die kantonalen Instanzen die Auffassung, die beanstandete Probe verstosse gegen das lebensmittelrechtliche Täuschungsverbot, weil sie den falschen Eindruck erwecke, das Bier werde in Luzern produziert. Demgegenüber machte die Beschwerdeführerin im Wesentlichen geltend, die Aufmachung ihres Produkts berge keine Täuschungsgefahr. Sie machte zudem geltend, sie vermarkte «nicht eine Biermarke mit geografischem Bezug zu Luzern (...), sondern ein Eigenbier mit ihrem Firmennamen [bzw. ein] Bier der Marke ,Lozärner Bier'» (E. 4).

\section{Lebensmittelrechtliche Grundlage}

10 Das Bundesgericht fasst zunächst die lebensmittelrechtliche Rechtslage $\mathrm{zu}-$ sammen (E. 4.1): Per 1. Mai 2017 (und damit während der Hängigkeit des kantonalen Rechtsmittelverfahrens) trat das Lebensmittelgesetz vom 20. Juni 2014 in Kraft. Gleichzeitig wurde das Bundesgesetz vom 9. Oktober 1992 über Lebensmittel und Gebrauchsgegenstände (AS 1995 1469; fortan: aLMG) aufgehoben (Art. 72 i.V.m. Anhang I LMG). Ebenfalls gleichzeitig wurde die lebensmittelrechtliche Ausführungsgesetzgebung umfang- reich angepasst: Die Lebensmittel- und Gebrauchsgegenständeverordnung vom 23. November 2005 (aLGV) wurde durch die gleichnamige Verordnung vom 16. Dezember 2016 ersetzt (LGV; Art. 94 Abs. 1 i.V.m. Art. 96 LGV; SR 817.02). Aufgehoben wurde auch die Verordnung des EDI vom 29. November 2013 über alkoholische Getränke (AS 2013 4977; fortan: aGetränkeverordnung). An ihre Stelle trat die Getränkeverordnung vom 16. Dezember 2016 (Art. 161 Ziff. 2 i.V.m. Art. 162 Getränkeverordnung).7 Zudem wurde die Verordnung des EDI vom 23. November 2005 über die Kennzeichnung und Anpreisung von Lebensmitteln (AS 2005 6159; fortan: aLKV) ausser Kraft gesetzt. Sie wurde in die Verordnung des EDI vom 16. Dezember 2016 betreffend die Information über Lebensmittel (LIV; SR 817.022.16) überführt (Art. 44 i.V.m. Art. 46 LIV).

11 Das Bundesgericht stellt sodann fest, dass das per 1. Mai 2017 in Kraft getretene LMG für die hier strittigen Fragen keine übergangsrechtlichen Regelungen (vgl. Art. 73 LMG) vorsieht. Dagegen würden Art. 95 Abs. 2 LGV (i.V.m. Art. 44 Abs. 1 und Art. 74 Abs. 2 LMG) eine «Übergangsfrist» von vier Jahren ab Inkrafttreten der Verordnung für die Kennzeichnung und Werbung von Lebensmitteln vorsehen. Nach Ablauf der Übergangsfrist dürfen nach altem Recht zusammengesetzte und gekennzeichnete Lebensmittel noch bis zur Erschöpfung der Bestände an die Konsumentinnen und Konsumenten abgegeben werden. Die Kennzeichnung und Werbung hat umfassend entweder dem neuen oder dem

7 Verordnung des EDI über Getränke vom 16. Dezember 2016 (SR 817.022.12). 
alten Recht zu entsprechen. ${ }^{8}$ Das auf bereits hängige Verfahren anwendbare Recht werde auch durch Art. 95 Abs. 2 LGV nicht näher bestimmt.

«Lozärner Bier Lager» falle in den sachlichen Anwendungsbereich sowohl der neuen als auch der alten Lebensmittelgesetzgebung. 9 Sowohl das alte wie das neue LMG würden unter anderem den Schutz der Konsumentinnen und Konsumenten vor Täuschungen im Zusammenhang mit Lebensmitteln bezwecken (Art. 1 lit. c LMG/Art. 1 lit. c aLMG) und «nahezu wortgleich» (E. 4.2.2) das lebensmittelrechtliche Täuschungsverbot in Art. 18 regeln: «Danach müssen sämtliche Angaben über Lebensmittel den Tatsachen entsprechen (Art. 18 Abs. 1 LMG/Art. 18 Abs. 1 aLMG). Ihre Aufmachung, Kennzeichnung und Verpackung sowie die Werbung für sie bzw. ihre Anpreisung dürfen die Konsumentinnen und Konsumenten nicht täuschen (Art. 18 Abs. 2 LMG/Art. 18 Abs. 2 aLMG). Unter anderem werden nach Art. 18 Abs. 3 aLMG Angaben und Aufmachungen als täuschend qualifiziert, wenn sie geeignet sind, beim Konsumenten falsche Vorstellungen über die Herkunft des Lebensmittels $\mathrm{zu}$ wecken. In ähnlicher Weise bestimmt Art. 18 Abs. 3 LMG, dass jene Aufmachungen, Verpackungen und Werbungen als täuschend qualifiziert werden, die geeignet sind, bei den Konsumentinnen falsche Vorstellungen über Produktionsland, Herkunft der Rohstoffe oder Bestandteile zu wecken.» (E. 4.2.2).

8 Das Bundesgericht verweist dazu auf die Erläuterungen des BLV zur LGV, S. $31 \mathrm{f}$.

9 Art. 3 Abs. 1 und Abs. 3 aLMG und Art. 4 Abs. 1 lit. $\mathrm{r}$ aLGV sowie Art. 1 Abs. 1 lit. d und Art. $41 \mathrm{ff}$. aGetränkeverordnung; Art. 4 Abs. 1 und Abs. 2 lit. a LMG sowie Art. 1 Abs. 1 lit. g Ziff. 1 und Art. 63 ff. der Getränkeverordnung.
13 Verboten seien «ungeachtet des diesbezüglich zu engen Wortlauts von Art. 18 Abs. 3 LMG» (E. 4.2.3) «nicht nur täuschende Angaben hinsichtlich des Produktionslands, sondern auch irreführende Aufmachungen im Hinblick auf die übrige (gegebenenfalls regionale oder örtliche) Herkunft eines Lebensmittels» (Art. 12 Abs. 1 LGV, unverändert übernommen aus Art. 10 Abs. 1 aLGV). Das Bundesgericht stellt sodann, auch mit Verweis auf die Materialien, fest, dass die lebensmittelrechtlichen Vorgaben zum Täuschungsverbot mit Ausnahme von Hinweisen auf Bestimmungen MSchG im alten und neuen Lebensmittelrecht nach Zweck und Wortlaut weitgehend übereinstimmen.

\section{Verhältnis des Lebensmittelrechts zum Herkunftskennzeichenrecht}

14 Das Bundesgericht hält sodann fest, dass sich im neuen LMG ein ausdrücklicher Hinweis auf das Markenrecht (präziser wäre wohl «auf das Recht der Herkunftskennzeichen») findet. Nach Art. 18 Abs. 2 Satz 2 LMG bleiben die Bestimmungen des MSchG über Angaben zur schweizerischen Herkunft vorbehalten. Analog dazu verbietet Art. 12 Abs. 2 lit. g LGV Hinweise, die geeignet sind, bei den Konsumentinnen und Konsumenten falsche Vorstellungen über die Herkunft eines Lebensmittels im Sinne des Markenschutzgesetzes zu wecken.

15 Das Bundesgericht betont weiter mit Verweis auf die Gesetzgebungsmaterialien und die spärliche Lehre, es stelle trotz erst kürzlich erfolgter Einfügung des Verweises auf das MSchG keine Neuerung dar, dass die Bestimmungen des Markenschutzgesetzes über die Herkunftsangaben beim Vollzug der Lebensmittelgesetz- 
gebung zu beachten sind. Es analysiert sodann, soweit für den Sachverhalt relevant, die während der Rechtshängigkeit des vorliegenden Verfahrens erfolgte Revision des MSchG. Während das bis 31. Dezember 2016 geltende Recht den Schutz geografischer Herkunftsangaben in wenigen Generalklauseln geregelt hatte (vgl. aArt. 47 ff. MSchG, AS 1993 274), wurden mit der am 1. Januar 2017 in Kraft getretenen Revision detaillierte Herkunftskriterien eingeführt. Welchen Kriterien ein Lebensmittel im Sinne des Lebensmittelgesetzes entsprechen muss, damit es mit einer Herkunftsangabe gemäss Art. 47 Abs. 1 MSchG versehen werden darf, bestimmt sich im neuen Recht nach Art. 48b MSchG (vgl. Art. 47 Abs. 3 lit. a i.V.m. Art. 48 Abs. 1 und Art. 48b Abs. 1 MSchG). Relevant sind weiter die Art. 52a Abs. 2 der Verordnung vom 23. Dezember 1992 über den Schutz von Marken und Herkunftsangaben (Markenschutzverordnung, MSchV; SR 232.111) sowie die Verordnung vom 2. September 2015 über die Verwendung von schweizerischen Herkunftsangaben für Lebensmittel (HasLV; SR 232.112.1), die gleichzeitig mit den revidierten Bestimmungen des MSchG am 1. Januar 2017 in Kraft getreten sind.

Das Bundesgericht erörtert, ob sich - wie von der Beschwerdeführerin suggeriert mit der Revision des MSchG an der parallelen Anwendbarkeit lebensmittelund kennzeichenrechtlicher Normen etwas geändert hat. Der neue Vorbehalt in Art. 18 Abs. 2 LMG zugunsten der Bestimmungen des MSchG über Angaben zur schweizerischen Herkunft sei ausdrücklich «nicht so zu verstehen, dass dem lebensmittelrechtlichen Täuschungsverbot kein eigenständiger Anwendungsbereich verbleibt, sobald ein Lebensmittel mit einer Herkunftsangabe im Sinne von Art. $47 \mathrm{ff}$. MSchG versehen ist. Die Verpflichtung zur Angabe des Produktionslands gemäss Art. 12 Abs. 1 lit. a LMG dürfe zwar nicht in einer Weise kennzeichenmässig umgesetzt werden, die den Vorschriften des 2. Titels des MSchG widerspricht» (E. 4.2.4.2). «Ebensowenig erlauben aber die Art. 47 ff. MSchG unter Ausblendung lebensmittelrechtlicher Bestimmungen eine Aufmachung von Lebensmitteln, die bei den Konsumenten tatsachenwidrige Vorstellungen über deren Herkunft wecken (vgl. Art. 18 Abs. 1 und Abs. 3 LMG/Art. 18 Abs. 1 und Abs. 3 aLMG)» (E.4.2.4.2), auch wenn Art. 52c MSchV und Art. 5 Abs. 1 HasLV je nach Auslegung sogar eine Konsumententäuschung in Kauf nehmen würden. Es gebe auch keinen Grund für die Annahme, der Gesetzgeber habe mit der Revision des 2. Titels des MSchG eine Lockerung des lebensmittelrechtlichen Täuschungsverbots beabsichtigt.

\section{Europäisches Lebensmittelrecht als Auslegungshilfe}

17 Das neue LMG orientiert sich am entsprechenden Recht der EU. Das Bundesgericht zieht deshalb ausdrücklich die entsprechenden Erlasse der EU und die dazu ergangene Rechtsprechung des Europäischen Gerichtshofs (EuGH) als Auslegungshilfe für das seit 1. Mai 2017 in Kraft stehende Lebensmittelrecht bei, soweit die Regelungen inhaltlich übereinstimmen. 
Aus diesem EU-Recht ${ }^{10}$ und aus der Rechtsprechung des EuGH ${ }^{11}$ ergebe sich, dass zur Beurteilung der Frage, ob eine Angabe zu einem Lebensmittel irreführend sein kann, hauptsächlich auf die mutmassliche Erwartung eines normal informierten, angemessen aufmerksamen und verständigen Durchschnittsverbrauchers in Bezug auf den Ursprung, die Herkunft und die Qualität des Lebensmittels abzustellen sei. Dabei komme es hauptsächlich darauf an, dass der Verbraucher nicht irregeführt und nicht $\mathrm{zu}$ der irrtümlichen Annahme verleitet wird, dass das Erzeugnis einen anderen Ursprung, eine andere Herkunft oder eine andere Eigenschaft als in Wirklichkeit hat. Die Angleichung des innerstaatlichen Lebensmittelrechts an jenes der EU habe somit nicht zu einer Absenkung des lebensmittelrechtlichen Täuschungsschutzes in der Schweiz führen sollen.

Art. 1 Abs. 1, Art. 5 Abs. 1, Art. 8 Abs. 1 lit. a und lit. c sowie Art. 16 der Verordnung [EG] Nr. 178/2002 des Europäischen Parlaments und des Rates vom 28. Januar 2002 zur Festlegung der allgemeinen Grundsätze und Anforderungen des Lebensmittelrechts, zur Errichtung der Europäischen Behörde für Lebensmittelsicherheit und zur Festlegung von Verfahren zur Lebensmittelsicherheit, Abl. L. 031 vom 1. Februar 2002, S. 1 ff.); Art. 2 Abs. 1 lit. a Ziff. i der Richtlinie 2000/13/EG des Europäischen Parlaments und des Rates vom 20. März 2000 zur Angleichung der Rechtsvorschriften der Mitgliedstaaten über die Etikettierung und Aufmachung von Lebensmitteln sowie die Werbung hierfür, Abl. L. 109 vom 6. Mai 2000, S. 29 ff. (per 13. Dezember 2014 aufgehoben gemäss Art. 53 Abs. 1 und neu gefasst in Art. 7 Abs. 1 sowie Art. 9 Abs. 1 und Art. 26 der Verordnung [EU] Nr. 1169/2011 des europäischen Parlaments und des Rates vom 25. Oktober 2011 betreffend die Information der Verbraucher über Lebensmittel [...], Abl. L. 304 vom 22. November 2011, S. $18 \mathrm{ff}$.).

11 Urteil des EuGH C-446/07 vom 10. September 2009 (Alberto Severi gegen Regione Emilia Romagna), Slg. 2009 I-08041 Rn. 61.
19 Mit Bezug auf den konkreten Fall schliesst das Bundesgericht daraus, es mache keinen Unterschied, ob die Rechtmässigkeit der angeordneten Massnahme nach neuem oder altem Recht und der dazu ergangenen Rechtsprechung beurteilt wird. Sowohl nach Art. 18 LMG wie nach Art. 18 aLMG verstossen Aufmachungen, die geeignet sind, beim Konsumenten falsche Vorstellungen über die Herkunft eines Lebensmittels zu wecken, etwa durch Angaben, die nicht den Tatsachen entsprechen, gegen das Täuschungsverbot.

20 Geradezu lehrbuchmässig fasst das Bundesgericht sodann die Grundsätze dieses Täuschungsschutzes zusammen (E. 4.3):

a) Täuschend sind namentlich unzutreffende Hinweise auf die Herkunft eines Lebensmittels oder Angaben, die tatsachenwidrig den Eindruck erwecken, das Produkt oder seine Ausgangsstoffe stammten aus einer bestimmten Gegend.

b) Eine Täuschung des Konsumenten kann auch durch wahre Angaben über ein Lebensmittel erfolgen, so wenn z.B. der Eindruck erweckt wird, dass es über besondere Eigenschaften verfügt, obwohl alle vergleichbaren Lebensmittel dieselben Eigenschaften aufweisen.

c) Ob die Aufmachung eines Lebensmittels als täuschend zu qualifizieren ist, hängt immer von verschiedenen Faktoren ab.

d) Ein Verstoss gegen das Täuschungsverbot kann sich aus einzelnen Angaben über das Lebensmittel ergeben, gegebenenfalls aber auch erst aus seinem gesamten Erscheinungsbild. 
e) Massstab zur Beurteilung, ob die Aufmachung eines Lebensmittels als täuschend im Sinne der genannten Bestimmungen zu qualifizieren ist, bildet der durchschnittliche Konsument; entscheidend ist dessen legitimes Informationsbedürfnis.

f) Dass der durchschnittliche Konsument die lebensmittelrechtlichen Vorschriften kennt, kann dabei nicht vorausgesetzt werden.

g) Es genügt die objektive Eignung zur Täuschung; der Nachweis, dass eine gewisse Zahl an durchschnittlichen Konsumenten tatsächlich getäuscht wurde, ist dazu nicht erforderlich.

h) Die entfernte Möglichkeit, dass das Produkt bei durchschnittlichen Konsumenten $\mathrm{zu}$ falschen Vorstellungen führt, reicht für einen Verstoss gegen das Täuschungsverbot nicht aus.

\section{Entscheid in der Sache}

21 Mit Bezug auf die Beurteilung von «Lozärner Bier» folgt das Bundesgericht der Vorinstanz. Das beanstandete Produkt weise in verschiedener Hinsicht enge Bezüge zum geografischen Raum Luzern her und sei damit objektiv geeignet, beim durchschnittlichen Konsumenten entsprechende Vorstellungen über die Herkunft des Produkts zu wecken, die mit den tatsächlichen Verhältnissen nicht übereinstimmen: Die Hinweise auf Luzern erfolgten nach der Sprechweise im lokalen Dialekt («Lozärn»); dies suggeriere eine erhöhte Authentizität dieser örtlichen Bezugnahme. In die gleiche Richtung wiesen die Firmenbezeichnung auf der Rückseite der Dose mitsamt der Adresse. Gleichenorts werde auf eine Webseite unter der URL «lozärner- bier.ch» verwiesen. Und schliesslich übernehme die Dose weitgehend die Gestaltung des Wappens des Kantons Luzern. Diese Gestaltungselemente stellen das Produkt nach der Beurteilung des Bundesgerichts in einen engen Bezugsrahmen zum geografischen Raum «Luzern» und vermitteln dem durchschnittlichen Konsumenten den Eindruck, das «Lozärner Bier Lager» stamme tatsächlich aus dieser Gegend.

Der auf der Rückseite des beanstandeten Produkts angebrachte Hinweis «hergestellt und abgefüllt in der Schweiz» sowie der stilisierte Umriss der Schweizerischen Eidgenossenschaft änderten daran nichts. Die übrigen Aufmachungselemente des beanstandeten Produkts bewirkten beim Konsumenten objektiv keine Veranlassung davon auszugehen, dass das Bier von irgendwoher aus der Schweiz stammen könnte. Da ein deutlicher Hinweis auf eine andere Herkunft fehle, wecke das Produkt beim Konsumenten in seinem gesamten Erscheinungsbild klar die Vorstellung, seine charakteristischen Eigenschaften seien ihm im Raum Luzern verliehen worden. Da dieser Eindruck nicht den Tatsachen entspreche, qualifiziert das Bundesgericht die Aufmachung als täuschend. Dass die Rezeptur und das Marketingkonzept behaupteterweise aus Luzern stammen, vermöge daran nichts zu ändern. Denn bei Bier sei für den Konsumenten der Produktionsort das massgebende Kriterium. Auch der Ort des Vertriebs, des Marketings oder der Verpackung seien nicht relevant, da diese Tätigkeiten dem Bier kein charakteristisches Gepräge zu verleihen vermögen.

23 Das Bundesgericht weist die Beschwerde gestützt auf diese Begründung $a b$ und 
setzt der Beschwerdeführerin eine neue Frist für die Bekanntgabe der Massnahmen zur Behebung des gesetzwidrigen Zustands an die DILV.

\section{Würdigung und drei Erkenntnisse}

24 Es bereitet keinerlei Mühe, das Ergebnis des Entscheids zu teilen. Auffällig ist immerhin der vom Bundesgericht betriebene Begründungsaufwand. Dieser hängt wohl damit zusammen, dass die von Sachverhalt und Beschwerdeführerin aufgeworfenen Rechtsfragen sich als nicht alltäglich erweisen.

Mit Bezug auf die Gehörsfrage bewegt sich der Entscheid zwar im Rahmen traditioneller und bewährter Rechtsprechung. Mutmassliche Parteien künftiger Verfahren und ihre Vertreter sollten sich indes in Erinnerung rufen, dass der Gehörsanpruch - insbesondere wie vorliegend in Fällen, in welchen die Parteien eine grosse Nähe zu einem Rechtsgebiet aufweisen - nicht bewirkt, dass sie von den Behörden ausdrücklich zu Stellungnahmen zu allfälligen Rechtsänderungen eingeladen werden müssen. Das Bundesgericht ermuntert solche Parteien indes gleichzeitig, in solchen Fällen aus eigener Initiative entsprechende Stellungnahmen einzureichen. Solche werden konsequenterweise von den Behörden in Zukunft auch nicht als irrelevant aus dem Recht gewiesen werden können.

\section{Verhältnis zwischen MschG und LMG}

26 Die erste und wichtigste Erkenntnis aus diesem Entscheid betrifft das Verhältnis zwischen MSchG und LMG. Als Auslegungsergebnis für dieses Verhältnis steht mit dem Entscheid Folgendes fest: a) Lebensmittelrechtlich verpflichtende deklaratorische Angaben (z.B. das Produktionsland) müssen auch die Vorschriften von Art. $47 \mathrm{f}$. MSchG einhalten. Eine lebensmittelrechtlich zutreffende, kennzeichenrechtlich (aufgrund der tatsächlichen Konsumentenwahrnehmung) aber unzutreffende Angabe müsste also so auf einer Verpackung angebracht werden, dass sie nicht kennzeichenmässig im Sinn des MSchG wirkt (sondern eben nur im sog. «Kleingedruckten», als reine Deklaration).

b) Umgekehrt dürfen Lebensmittel obwohl die Herkunftskriterien nach Art. 47 f. MSchG dies allenfalls sogar erlauben würden, nicht so aufgemacht und gekennzeichnet werden, dass Konsumenten getäuscht werden, weil bei ihnen aufgrund der Kennzeichnung tatsachenwidrige Vorstellungen geweckt werden.

27 Der vorliegende Fall illustriert dies musterhaft: Das Institut für Geistiges Eigentum (IGE) hatte die Eintragung von «Lozärner Bier» fig. als Marke für Bier schweizerischer Herkunft (nicht lediglich luzernischer Herkunft), d.h. als Registerrecht akzeptiert und damit eine Täuschungsgefahr nach Art. 2 Bst. c MSchG im Markeneintragungsverfahren verneint. Dennoch greift hier das lebensmittelrechtliche Täuschungsverbot aufgrund der konkreten Warenaufmachung mit zusätzlichen Merkmalen (farbliche Gestaltung, Web-Adresse etc.).

\section{Gesamteindruck}

28 Die zweite Erkenntnis aus diesem Entscheid ist eine Bestätigung langjähriger kennzeichenrechtlicher Grundsätze: 
Massgebend ist jeweils der Gesamteindruck einer Produktgestaltung (in den Worten des Bundesgerichts «gesamtes Erscheinungsbild») in Bezug auf den Ursprung, die Herkunft und die Qualität des Lebensmittels. Relevant ist dabei der Gesamteindruck beim Durchschnittskonsumenten. Dieser entspricht, das ergibt sich aus dem auslegungsweise herangezogenen europäischen Lebensmittelrecht, grundsätzlich dem europäischen Verbraucherleitbild, d.h. dem normal informierten, angemessen aufmerksamen und verständigen Durchschnittsverbraucher.

Dieser Gesamteindruck kann allenfalls, wie das Bundesgericht auch anklingen lässt, durch deutliche Hinweise auf eine andere als die lebensmittelrechtlich unzulässige Herkunft kompensiert werden. Diese Kompensationsoption durch entlokalisierende Zusätze entspricht der Rechtsprechung des deutschen Bundesgerichtshofs (BGH) in den sog. Warsteiner-Fällen ${ }^{12}$. Dort wurde anerkannt, dass in gewichtigen Ausnahmesituationen schutzwürdige Interessen gegenüber einem auf eine unrichtige geographische Herkunftsangabe gestützten Kennzeichnungsverbot bestehen können, die durch entlokalisierende, die Täuschungsgefahr ausschliessende Zusätze beseitigt werden können.

\section{Charakteristische Eigenschaft}

3o Die dritte Erkenntnis aus diesem Entscheid ist die entscheidende Funktion der «charakteristischen Eigenschaften» eines Produkts aus der Sicht der Konsumenten. Bei Bier geht das Bundesgericht davon aus (möglicherweise im Sinne eines Erfahrungssatzes, dies erschliesst sich aus dem Entscheid nicht), sei dies der Brauort und nicht der Ort der Rezepturkomposition, der Vertriebslogistik oder des Entwurfs der Marketingstrategie.

31 Ob dies bedeutet, dass auch Wasser und Hopfen luzernischer Herkunft sein müssen, weil diese Bestandteile neben dem Brauort möglicherweise die Konsumentenerwartung prägen, wird im Entscheid nicht thematisiert und wäre nach Art. 48b MSchG zu prüfen.

12 Warsteiner II und III, I ZR 54/96 und 55/96. 